\title{
BRDF Estimation of Structural Color Object by Using Hyper Spectral Image
}

\author{
Yoshie Kobayashi \\ The University of Tokyo \\ Tokyo, Japan \\ yoshiedcvl.iis.u-tokyo.ac.jp \\ Imari Sato \\ National Institute of Informatics \\ Tokyo, Japan \\ imarik@nii.ac.jp
}

\author{
Tetsuro Morimoto \\ Toppan Printing Co., Ltd \\ Tokyo, Japan \\ tetsuro.morimoto@toppan.co.jp \\ Yasuhiro Mukaigawa \\ Osaka University \\ Osaka, Japan \\ mukaigaweam.sanken.osaka-u.ac.jp
}

\author{
Katsushi Ikeuchi \\ The University of Tokyo \\ Tokyo, Japan \\ ki@cvl.iis.u-tokyo.ac.jp
}

\begin{abstract}
In computer graphics, there are several methods to render thin film interference effects, but it sets the parameters of interference manually. To reconstruct more accurate appearance of the thin film, we need to estimate the BRDF parameters of the thin film, the refractive index and film thickness. In this paper, we propose a novel method for estimating the BRDF of the thin film interference and representing the appearance accurately. Using peak wavelengths which enhance the spectral intensities, we estimate the refractive index and film thickness separately, and then it reduces the computational time of estimation comparied with the steepest descent method. In the experiments, we evaluate the accuracy of estimated parameters and reconstructed BRDF to show the effectiveness of our method.
\end{abstract}

\section{Introduction}

It is important to acquire and reconstruct the appearance of real world objects in graphics and computer vision. It benefits many applications, such as movies, games, and digitizing cultural heritages. There are many materials with complex reflection properties, such as scattering, absorption, diffraction, refraction and interference. It is difficult to acquire and represent their appearance. Among these reflection properties, the interference has very complex reflection effects. The color appearance is changed depending on not only the view direction but also the light direction. This color change is caused by a physical phenomenon, not by a pigment, and it is so called structural color.

In this paper we focus on the interference of thin film with two layers. There are many objects with an interference reflection, such as laminated material, soap bubbles and oil film. Our goal is to estimate the BRDF of thin film interference and represent the appearance accurately. Using a hyper-spectral camera, we can estimate the BRDF at all pixels even if the BRDF is spatially inhomogeneous.

The estimation of the BRDF of thin film interference can be applied to many fields, such as industries, biology, archeology, and medicine. For example, in computer graphics we can render soap bubbles more accurately. Furthermore, in industrial fields we can estimate the distribution of diffused oil. In factory design we can confirm the realistic appearance of new coating products in digital space.

The interference effect is caused by the phase difference between incident and outgoing light waves on the top layer of the thin film. The phase difference is dependent on the refractive index and optical thickness of the top layer. Hence, we acquire the BRDF of the thin film by estimating the refractive index and optical thickness. For this task, we propose a novel method to rapidly estimate the BRDF in each pixel.

The rest of the paper is organized as follows. Section 2 introduces the methods for acquiring and estimating BRDF and estimating the refractive index and film thickness. Section 3 introduces a reflectance model of thin film interference. Section 4 explains a method for estimating the refractive index and film thickness. In section 5, we evaluate 
the proposed method with some evaporated metal films and discuss the estimation error in the experiment. In section 6 , we summarize our paper.

\section{Related Work}

Currently there are many states of the art techniques to acquire accurate reflection properties of objects and to represent their appearances. BRDF represents the color change along with light and view directions. The BRDF acquisition approach can be divided into three categories.

The first approach is based on gonioreflectometers which control the illumination and view directions by the sequential and mutual positioning of the light, sensor, and sample $[1,2]$. However it takes a lot of time to acquire the reflectance properties of the structural color object. The second approach uses mirrors $[3,4]$. This approach can rapidly acquire BRDF, but due to the high dimensionality of the acquired BRDF, it is difficult to represent the accurate appearance of the object. The final approach is proposed by Sato et. al. [5]. They represent the object appearance using the image-based approach, but it can represent only the visualization.

To solve these problems, various BRDF models $[6,7$, $8,9]$ were proposed to represent high dimensional data in more compact way. Dong et. al. [10] developed a new hand-held BRDF capturing device, and acquired the spatially varying BRDF (SVBRDF) using the boosting technique. However, these methods cannot represent the accurate color change of thin film by the interference effect because they don't consider the optical path difference of the thin film. Morimoto et. al. [11] estimated the optical thickness and opacity of layered surfaces, and represented the appearance of layered surfaces of arbitrary thickness. However, this method cannot handle the interference effect of the thin film.

Various rendering methods have been proposed to describe the color change of a structural color object. Iwasaki et. al. [12] rendered soap bubbles by using a physical model of thin film interference. Hirayama et. al. [13, 14] used the physics-based interference model of multi-layered film, and rendered eyeglasses and mother-of-pearl. Modeling the microstructure of CDs, Sun et. al. [15][16]rendered them more realistically. Sadeghi et. al. [17] calculated the refracted ray in moisture by ray tracing, and then represented the rainbow more correctly. Furthermore, Cuypers et. al. [18] showed that the Wigner distribution function can represent the BRDF of a diffraction grating and it is as accurate as the physics model. These rendering methods manually set the parameters of physical models, therefore the estimation of these parameters is needed to represent the reflection properties of the structural color of real objects.

In the optics field, a method is proposed to estimate the refractive index and film thickness which are physical pa-

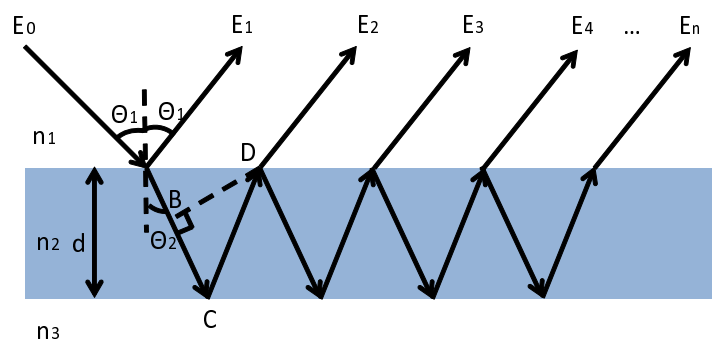

Figure 1. Schematic diagram of thin film interference considering multiple reflections. $n_{1}$ and $n_{2}$ are refractive index, $d$ is film thickness, $\theta_{1}$ is incident angle and $\theta_{2}$ is refracting angle.

rameters of thin film. One famous method is the interference spectroscopy [19] estimating the film thickness from peak wavelengths which enhance spectral intensities. Another method is the ellipsometry [20] using amplitude ratio and phase difference between perpendicular and parallel polarizations. These methods need the known refractive index and measure at only one point; it is difficult to estimate spatially varying film thickness with an unknown refractive index.

In this paper we propose a novel method for estimating the refractive index and film thickness which are the parameters of thin film BRDF. We focus on a peak wavelength which enhances spectral intensity and is equal to the integral multiple of the optical path difference. Therefore, at this wavelength we can define the refractive index and film thickness more easily. Furthermore, we use a hyper spectral camera and measure the reflectance as a spectral image.

\section{Thin-Film Interference Reflectance Model}

In optics, the reflectance model [21] of thin film interference is proposed. This model can represent the color change of the thin film interference along an incident angle by an optical path difference. The color change of the thin film only depends on the incident angle: the color change does not depend on the incident angle. This model can represent the color change of the thin film along the incident angle. That is to say this model is the BRDF of the thin film interference.

Considering the multi-path reflection in Fig. 1, the observed light at the point $E_{n}$ is represented as shown below.

$$
\begin{aligned}
E= & E_{1}+E_{2}+E_{3}+E_{4}+\cdots \\
= & E_{0}\left(r_{12}+t_{12} t_{21} r_{23} e^{i \Delta}+t_{12} t_{21} r_{23}^{2} r_{21} e^{2 i \Delta}\right. \\
& +t_{12} t_{21} r_{23}^{3} r_{21}^{2} e^{3 i \Delta}+\cdots \\
= & E_{0}\left(r_{12}+t_{12} t_{21} r_{23} e^{i \Delta}\left(1+r_{23} r_{21} e^{i \Delta}\right.\right. \\
& \left.\left.+r_{23}^{2} r_{21}^{2} e^{2 i \Delta}+\cdots\right)\right) \\
= & E_{0}\left(r_{12}+t_{12} t_{21} r_{23} e^{i \Delta} \frac{1}{1-r_{23} r_{21} e^{i \Delta}}\right)
\end{aligned}
$$

where $r_{12}, r_{23}$ and $r_{21}$ are Fresnel reflection coefficients, and $t_{12}$ and $t_{21}$ are Fresnel transmittance coefficients. $\varphi$ is 
the optical path difference which is the distance of $B C+$ $C D$ as shown in Fig. 1. $\Delta$ is the phase difference, and represented by Eq. (3).

$$
\begin{gathered}
\varphi=2 d n_{2} \cos \theta_{2} \\
\Delta=\frac{2 \pi \varphi}{\lambda}
\end{gathered}
$$

By using $r_{21}=-r_{12}$ and $t_{21} t_{12}+r_{12}^{2}=1$, the amplitude of reflection coefficients is defined by Eq. (4).

$$
\begin{aligned}
r & \equiv \frac{E}{E_{0}} \\
& =r_{12}+t_{12} t_{21} r_{23} e^{i \Delta} \frac{1}{1-r_{23} r_{21} e^{i \Delta}} \\
& =\frac{r_{12}-r_{12} r_{23} r_{21} e^{i \Delta}+\left(1-r_{12}^{2}\right) r_{23} e^{i \Delta}}{1-r_{23} r_{21} e^{i \Delta}} \\
& =\frac{r_{12}+r_{23} e^{i \Delta}}{1+r_{23} r_{12} e^{i \Delta}}
\end{aligned}
$$

The reflectance is given by the square of the absolute value of Eq. (4) as shown below in Eq. (5).

$$
R=|r|^{2}
$$

Fresnel reflection coefficients in Eq. (4) are defined for perpendicular ( $\mathrm{S}$-wave) and parallel (P-wave) polarizations by the refractive index, thickness and incident angle. When a ray strikes the interface between two media of refractive indices $n_{1}$ and $n_{2}$ in Fig. 1, Fresnel reflection coefficients are defined in Eq. (6) and (7). Fresnel reflection coefficients between two media of refractive indices $n_{2}$ and $n_{3}$ in Fig. 1 are defined in the same way as the following equations.

$$
\begin{aligned}
& r_{12}^{s}=\frac{n_{1} \cos \theta_{1}-n_{2} \cos \theta_{2}}{n_{1} \cos \theta_{1}+n_{2} \cos \theta_{2}} \\
& r_{12}^{p}=\frac{n_{2} \cos \theta_{1}-n_{1} \cos \theta_{2}}{n_{2} \cos \theta_{1}+n_{1} \cos \theta_{2}}
\end{aligned}
$$

Assigning Eq. (6), (7) and (4) to Eq. (5), Eq. (5) is represented by the refractive index, film thickness and incident angle. Eq. (5) describes the spectrum of the S-wave and P-wave along light and view directions: Eq (5) is the BRDF model of thin film interference. In this paper, we use Eq. (5) as a BRDF model of thin film interference. Therefore, obtaining the refractive index and film thickness, we can reconstruct the BRDF of thin film by using Eq. (5).

\section{BRDF Parameter Estimation}

As mentioned in the previous section, the refractive index and film thickness are needed to represent a thin film BRDF. Therefore we need to estimate the refractive index

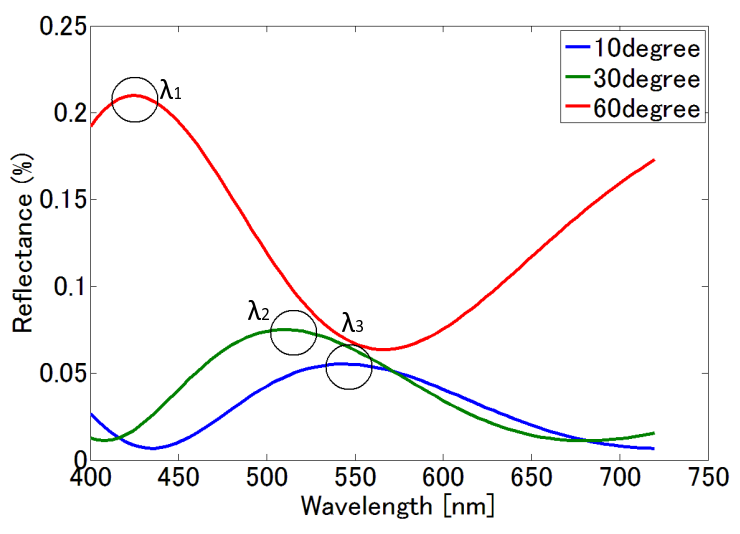

Figure 2. Reflectance of Thin Film Interference at 10, 30, and 60 degrees Wavelengths of areas enclosed by circles are peak wavelengths. The refractive index of this reflectance is 1.37 and the film thickness is $400 \mathrm{~nm}$. Blue, green, and red lines are reflectance at 10,30 , and 60 degree, respectively.

and film thickness from a measured reflectance. In this section, we propose a novel method for estimating these parameters.

In the estimation, we have three assumptions. First, the incident angle is known. As mentioned in Sec. 3, the parameters of the reflectance model are the refractive index, film thickness and incident angle. If all the incident angles are unknown, the number of parameters becomes larger than that of the measured reflectance, and then it is difficult to estimate. Second, the refractive indices are consistent at all reflectance image pixels. Third, the thin film thickness is different at each pixel but changes smoothly. To estimate the refractive index and film thickness more stably, we need to reduce the measurement noise, and then second and third assumptions are needed.

The steepest descent method is suitable to estimate the best combination of the refractive index and film thickness at once. There are numerous local minimums between the reflectance model and the measured reflectance. However, this method takes so much time since its order is $O\left(m n^{2}\right)$ where $m$ is the number of pixels, $n$ is the number of samples of the refractive index and film thickness.

Using the peak wavelength which enhances the spectrum intensity, we can estimate these parameters separately and reduce the computational cost. Fig. 2 shows an example of peak wavelengths enclosed by circles. At these wavelengths, the optical path difference is equal to the integral multiple. Furthermore, the peak wavelength gradually shifts to the shorter wavelength as the incident angle approaches 90 degrees. With these clues, we estimate by the following procedure.

Firstly, we estimate the refractive index with the peak wavelength which enhances the spectrum intensity. Secondly, we pick up the candidate of the film thickness with the estimated refractive index and determine by the least 
mean-square error between the reflectance model and the measured reflectance. The order of this method is $O(\mathrm{ml}) . l$ is the number of candidates of the film thickness.

\subsection{Refractive Index Estimation}

We introduce the estimation method for refractive index. Eq. (2) is the optical path difference and rewritten as Eq. (8) with incident angle $\theta_{1}$ by Snell's law.

$$
\varphi=2 d \sqrt{n_{2}^{2}-\sin ^{2} \theta_{1}}
$$

where $d$ is film thickness, $n_{2}$ is a refractive index and $\theta_{1}$ is an incident angle. It becomes integral multiple of the peak wavelength and rewritten as follows.

$$
m \lambda=\varphi
$$

Assuming refractive index and film thickness are consistent at two different angles, $\theta_{1_{1}}, \theta_{1_{2}}$, the optical path difference is integral multiple of the peak wavelengths shown in Eq. (10) and (11) with peak wavelengths.

$$
\begin{aligned}
& m \lambda_{1}=4 \pi d \sqrt{n^{2}-\sin ^{2} \theta_{1_{1}}} \\
& m \lambda_{2}=4 \pi d \sqrt{n^{2}-\sin ^{2} \theta_{1_{2}}}
\end{aligned}
$$

where $m$ is a natural number, $\theta_{1_{1}}$ and $\theta_{1_{2}}$ are incident angles, and $\lambda_{1}, \lambda_{2}$ are peak wavelengths. Combining Eq. (10) and (11), the refractive index is defined as Eq. (12).

$$
n_{2}=\sqrt{\frac{\lambda_{2}^{2} \sin ^{2} \theta_{1_{1}}-\lambda_{1}^{2} \sin ^{2} \theta_{1_{2}}}{\lambda_{2}^{2}-\lambda_{1}^{2}}}
$$

\subsection{Film Thickness Estimation}

We also introduce the estimation method for the film thickness with the estimated refractive index. By combining Eq. (10) and (11), the film thickness, $d$, is defined by Eq. (13).

$$
d=\frac{m \lambda_{1,2}}{2 \sqrt{n_{2}^{2}-\sin ^{2} \theta_{1_{1,2}}}}
$$

In Eq. (13), the natural number, $m$, is unknown; unless we determine the natural number, $m$, we are not able to get the film thickness. As shown in Eq. (14), we determine $m$ by minimizing the squared error between the measured reflectance and the reflectance model shown in Eq. (5).

$$
\left|R_{\text {model }}-R_{\text {measured }}\right| \underset{m}{\longrightarrow} \min
$$

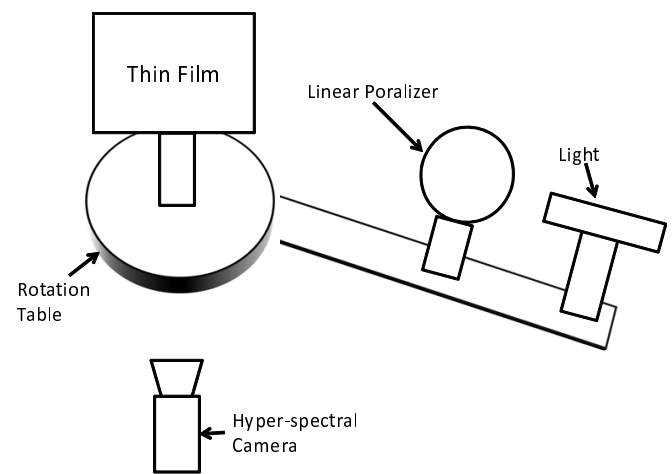

(a) Schematic Diagram of Setup

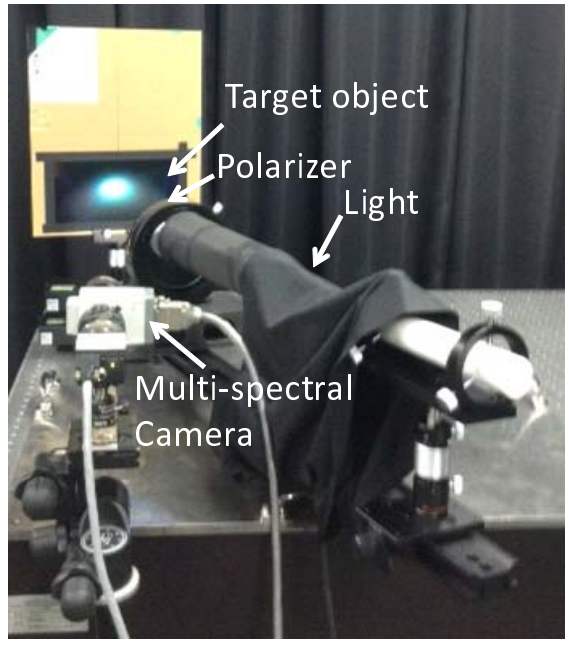

(b) Actual Setup

Figure 3. Experimental Setup for Measuring Reflectance of Thin Film (a) shows the schematic diagram of the setup. (b) shows the actual setup. The distance between light and thin film is $0.8[\mathrm{~m}]$. The distance between camera and thin film is $0.6[\mathrm{~m}]$.

\section{Experiment}

To evaluate the accuracy of the estimated BRDF, we conducted an experiment with thin films with known parameters. We investigated the accuracy of estimated refractive index and film thickness. Using the estimated parameters, we evaluate the color difference and RMSE along an incident angle. We also simulate the thin film appearance by $\mathrm{CG}$ and compare it with real object images.

\subsection{Experimental Setup}

The experimental setup is shown in Fig. 3. A target object and a light source are attached on the rotation table which adjusts an incident angle. The incident angle is changed from 10 to 50 degrees by 10 degrees. The reflectance of the thin film is measured by the hyper-spectral camera which transmits linearly polarized light. The hyperspectral camera is composed of Liquid Crystal Tunable Filter (vari spec cri) and monochrome camera. The LCTF can change its transmitted wavelength electrically. The view- 


\begin{tabular}{|l|c|c|c|c|c|c|}
\hline Target & $\begin{array}{c}\text { Ground truth of } \\
\text { refractive index }\end{array}$ & $\begin{array}{c}\text { Estimated } \\
\text { refractive index }\end{array}$ & $\begin{array}{c}\text { Ground truth of } \\
\text { thickness[nm] }\end{array}$ & $\begin{array}{c}\text { Estimated } \\
\text { thickness[nm] }\end{array}$ & BRDF error & RMSE \\
\hline Sample 1 & 1.370 & 1.374 & 400 & 370 & 0.72 & 0.04 \\
Sample 2 & 1.370 & 1.367 & 600 & 551 & 1.21 & 0.07 \\
Sample 3 & 2.370 & 2.674 & 500 & 541 & 5.63 & 0.18 \\
\hline
\end{tabular}

Table 1. Estimated refractive index and average film thickness of Fig. 5. The BRDF error is the average color difference among all incident angles at all pixels of Fig. 5. RMSE is average among all incident angles of 5.

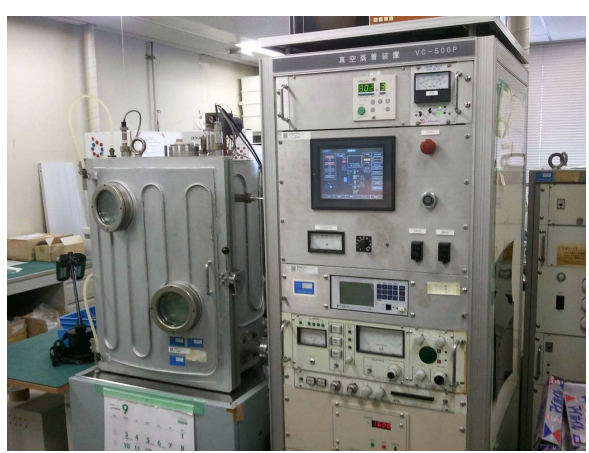

(a) Vacuum Coating Equipment

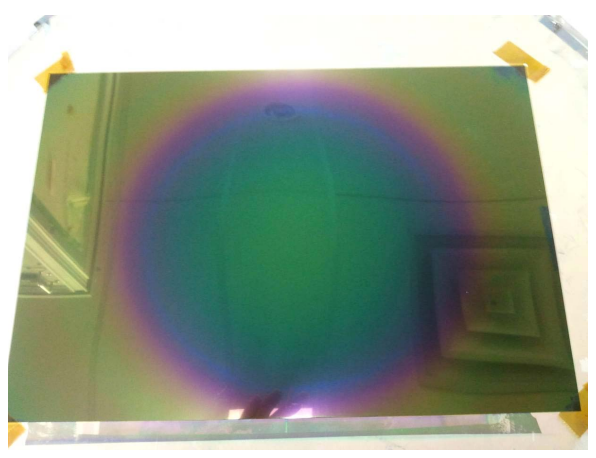

(b) $600 \mathrm{~nm} M g F_{2}$ in thickness

Figure 4. (a) Vacuum coating equipment to evaporate metal on PET film and (b) Evaporated film thickness of $M g F_{2}$ is $600 \mathrm{~nm}$.

ing angle of camera is about 30 degrees. The band width in this experiment is 4 [nm]. We also set a linear polarizer to transmit only S-wave, so the hyper-spectral camera observes only $\mathrm{S}$-wave reflectance.

As a target object, we use three samples of the thin film where the metal is evaporated onto the polyethylene terephthalate (PET) film.

- Sample 1 Material: $M g F_{2}$ Thickness: $400 \mathrm{~nm}$

- Sample 2 Material: $M g F_{2}$ Thickness: $600 \mathrm{~nm}$

- Sample 3 Material: $Z n S$ Thickness: $500 \mathrm{~nm}$

To evaporate these materials as the above film thickness, we use the vacuum coating equipment as shown in Fig. 4 (a). The vacuum coating equipment can control the thickness by the Quartz Crystal Microbalance. The example of evaporated thin film is shown in Fig. 4 (b).

\subsection{Evaluation}

Fig 5 (a), (c) and (e) are the distribution maps of estimated film thickness of each sample. We also show the error maps of estimated film thickness in Fig. 5 (b), (d) and (f). The error is calculated by $\left|d_{\text {true }}-d_{\text {estimated }}\right|$. In Table 1, the estimated refractive index and the average film thickness of Fig. 5 (a), (c) and (e) are shown. The ground truth of refractive index in Table 1 is acquired from the material information. Comparing these data the error of the refractive index is approximately 0.02 for Sample 1 and Sample 2. It becomes 0.30 for Sample 3. The error of average film thickness is about $30 \mathrm{~nm}$ and $50 \mathrm{~nm}$ for Sample 1 and Sample 2, respectively. It is about $40 \mathrm{~nm}$ for Sample 3.

Table 1 also shows the average color difference and RMSE among all incident angles of all reflectance image pixels. The color difference is defined as Eq. (15).

$$
\Delta E^{*} a b=\sqrt{\left(\Delta L^{*}\right)^{2}+\left(\Delta a^{*}\right)^{2}+\left(\Delta b^{*}\right)^{2}}
$$

Table 2 shows the levels of color differences; human eyes perceive how different two colors are. The difference of Sample 1 and Sample 2 is 0.72 and 1.21, respectively. This is slightly different as shown in Table 2. It becomes 5.63 for Sample 3, and this is an appreciable difference.

\begin{tabular}{|l|c|}
\hline Level of color difference & $\Delta E^{*} a b$ \\
\hline trace & $0 \sim 0.5$ \\
slight & $0.5 \sim 1.5$ \\
noticable & $1.5 \sim 3.0$ \\
appreciable & $3.0 \sim 6.0$ \\
much & $6.0 \sim 12.0$ \\
very much & over 12.0 \\
\hline
\end{tabular}

Table 2. Level of Difference or Distance between Two Colors

We also show the RMSE in Table 1. The RMSE is calculated by Eq. (16).

$$
R M S E=\sqrt{\left(R_{\text {true }}(\lambda)-R_{\text {estimated }}(\lambda)\right)^{2} / N}
$$

$N$ is the dimension of the reflectance spectra. The reflectance is not greater than 1.0. Therefore, the RMSE of Sample 1 is $4 \%$ and that of Sample 2 is $7 \%$. In case of Sample 3, it becomes $18 \%$.

\subsection{Rendering image}

We render CG images with the estimated refractive indices and film thickness in Table 1. The renderer is PBRT 


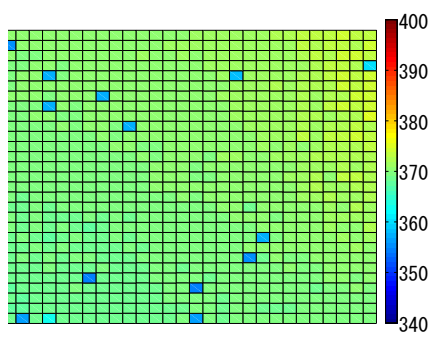

an

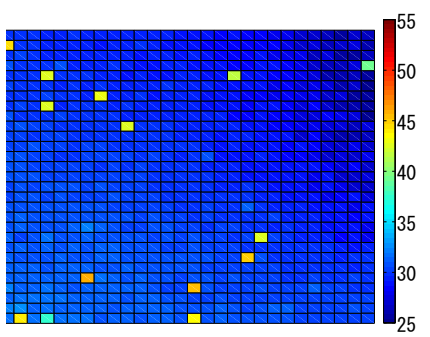

(d)

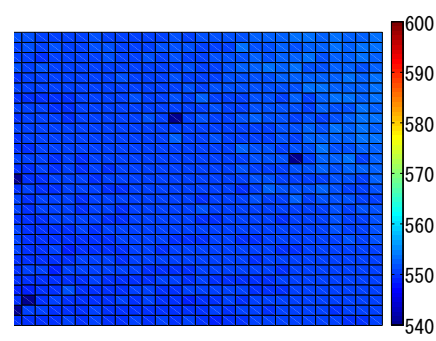

(h)

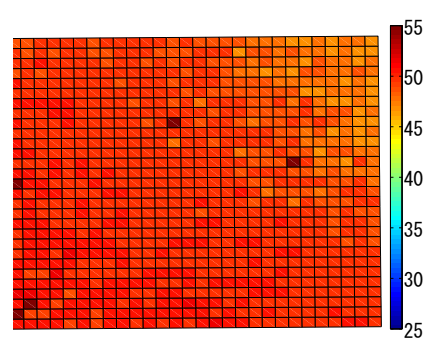

(e)

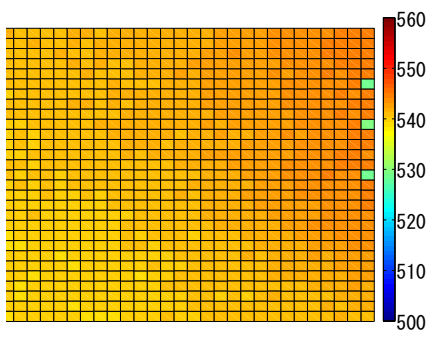

is

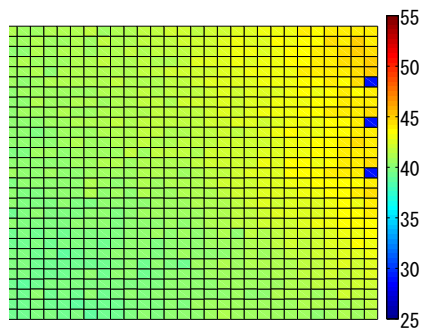

(f)

Figure 5. Images on upper side are distribution maps of the estimated film thickness of each target. Images on bottom side are the error maps of the estimated film thickness. (a) and (d) are distribution maps for Sample 1 (b) and (e) are distribution maps for Sample 2 (c) and (f) are distribution maps for Sample 3.

[22]. The right side images of Fig. 6 show rendering images. In these images we use environment map and Teapot as a rendering object. We also show the target object images on the left side of Fig. 6. Comparing the images on the left and right sides, the color change is very similar in the case of Sample 1 and Sample 2. The rendering image of Sample 3 is slightly different from the color of the real object because of the estimation error of BRDF parameters.

\subsection{Discussion}

In previous section, the film thickness error of Sample 2 is the largest. However, the color difference and RMSE are small enough among Sample 1 and Sample 2. Regarding Sample 3, the color difference is appreciable and RMSE becomes about $18 \%$. In this section, we discuss the reason for these errors.

Firstly, we discuss the reason why the color difference and RMSE are small enough in spite of the largest thickness error for Sample 2. The error of the color difference and RMSE is due to the optical path difference which causes the color change of the thin film interference. The optical path difference can be defined by the multiplication of the refractive index and film thickness. Comparing with the error of optical path difference, the error of Sample 2 is small enough because the error of the refractive index is small enough. Therefore, the color difference and RMSE of Sample 2 become smaller.

Secondly, we discuss the reason why the estimation error is so large for Sample 3. The larger estimation error for Sample 3 is due to narrow interval of the peak wave-

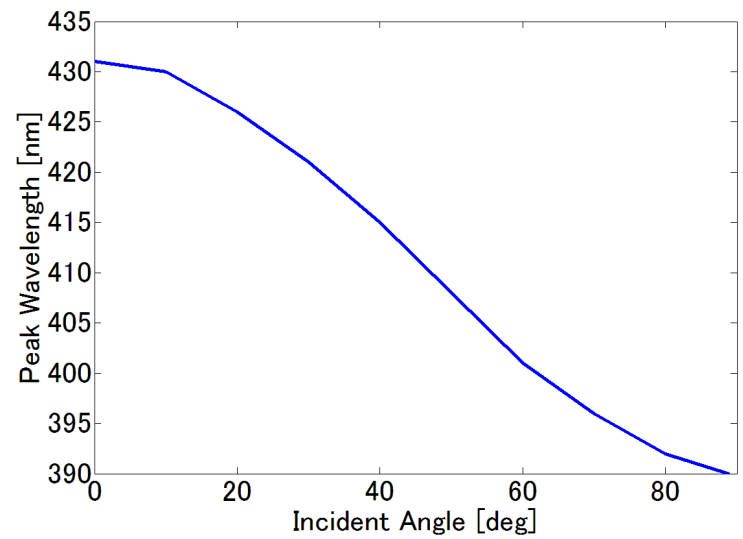

Figure 7. Peak Wavelength along Incident Angles. The film material is $Z n S$. The film thickness is $500 \mathrm{~nm}$.

lengths between nearby incident angles. Fig. 7 shows the peak wavelengths of Sample 3 along incident angles. As shown in Fig. 7, the interval between nearby incident angles by 10 degree is about $4.1 \mathrm{~nm}$. This interval is very close to the wavelength sampling rate by the hyper-spectral camera which is $4 \mathrm{~nm}$ in this experiment. Due to the measurement noise of the reflectance, it is difficult to detect the peak wavelength correctly, and then the estimation error of the refractive index and film thickness becomes large. Therefore the estimation error of the BRDF becomes appreciable.

The solution of this large estimation error is to use the hyper-spectral camera whose wavelength sampling rate is small enough compared with the interval of the peak wavelength between nearby incident angles. However, this solution shows the limitation of our method. From Eq. (18), the 


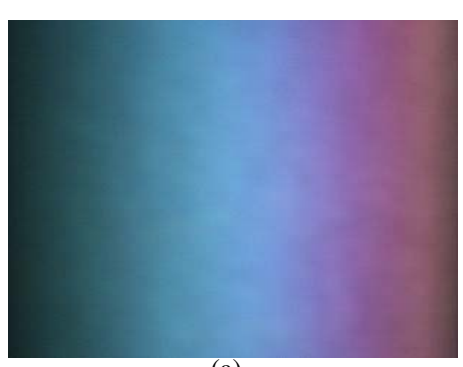

(a)

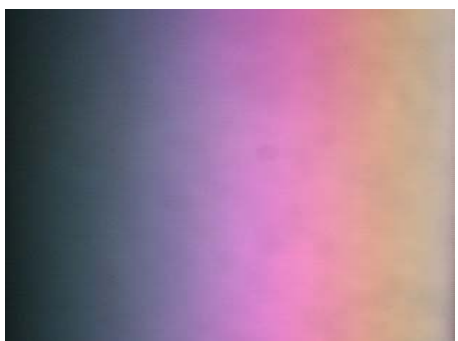

(c)

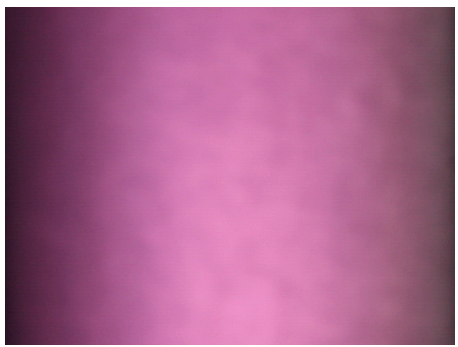

(e)

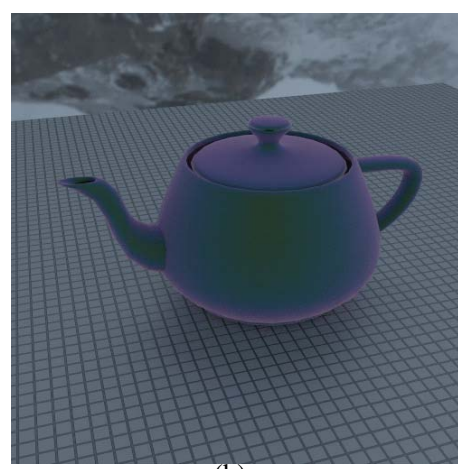

(b)

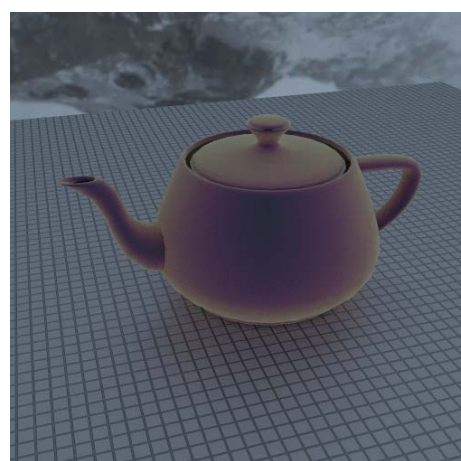

(d)

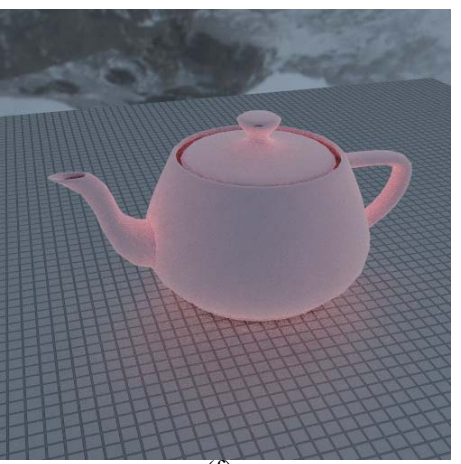

(f)

Figure 6. Left side of images are real objects on evaporated thin film. Right side images are rendered images with parameters estimated from real objects. (a) $M g F_{2} 400 \mathrm{~nm}$. (b) refractive index:1.374 and film thickness:370 nm (c) $M g F_{2} 600 \mathrm{~nm}$. (d) refractive index:1.367 and film thickness:551 nm (e) $Z n S 500 \mathrm{~nm}$. (f) refractive index:2.674 and film thickness:541 nm

scale of $\gamma$ greatly affects the interval of peak wavelengths between nearby incident angles. As shown in Eq. (17), the scale of $\gamma$ is almost determined by the refractive index. The larger the refractive index is, the smaller the rate of change of Eq. (17) is. Therefore, there is a limit to the refractive index with high estimation accuracy.

$$
\begin{gathered}
\gamma=\sqrt{n_{2}^{2}-\sin ^{2} \theta_{1}}-\sqrt{n_{2}^{2}-\sin ^{2} \theta_{2}} \\
\lambda_{1}-\lambda_{2}=\frac{2 d \gamma}{m}
\end{gathered}
$$

where $n_{2}$ is refractive index, $\theta_{1}$ and $\theta_{2}$ are incident angles. $\lambda_{1}$ and $\lambda_{2}$ are peak wavelength, $d$ is film thickness and $m$ is natural number.

\section{Conclusion}

This paper proposed a novel method for estimating BRDF of thin film interference and representing the appearance accurately. In the estimation of the refractive index, we focus on the peak wavelength where the optical path difference is equal to an integral multiple. With the peak wavelength, the refractive index can be defined in a compact way. Using the peak wavelength and the estimated refractive index, we can narrow down the candidates, and easily estimate the film thickness with them. The experiment shows that the accuracy of the proposed method is adequate and it can represent the color change of the thin film well. The 
results also shows the limitation of our method that we can not estimate with high accuracy with larger refractive index which make the interval of the nearby peak wavelength smaller than wavelength sampling rate.

\section{Acknowledgment}

The authors would like to thank Dr. Patricia Knapp for proofreading English manuscript. This research has been supported by the Japan Society for the Promotion of Science (JSPS) through the "Funding Program for Next Generation World-Leading Researchers (NEXT Program)," initiated by the Council for Science and Technology Policy (CSTP).

\section{References}

[1] M. Holroyd, J. Lawrence, and T. Zickler, "A coaxial optical scanner for synchronous acquisition of 3D geometry and surface reflectance," ACM Transactions on Graphics.,, pp. 1-12, 2010.

[2] K.J. Dana, B. van Ginneken, S.K. Nayar, and J.J. Koenderink, "Relectance and texture of real world surfaces," ACM Transactions on Graphics., , Vol 18(1)pp. 1- 34, 1999.

[3] Y.Mukaigawa, K.Sumino, Y.Yagi, "Rapid BRDF Measurement using an Ellipsoidal Mirror and a Projector", IPSJ Transactions on Computer Vision and Applications., Vol.1, pp.21-32, Jan.2009.

[4] K.J. Dana,"BRDF/BTF Measurement Device," In ICCV 2001., volume2, pp460- 466, July 2001

[5] I. Sato, T. Okabe, Y. Sato, "Appearance Sampling of Real Objects for Variable Illumination, h Int. J. Computer Vision., 2007.

[6] J. H. Lambert, "Photometria sive de mensure de gratibus luminis, colorum umbrae." Eberhard Klett, 1760.

[7] G. Ward. "Measuring and modeling anisotropic reflection," ACM SIGGRAPH Computer Graphics, 26(2):265-272, 1992.

[8] K. Torrance and E. Sparrow. "Theory for off-specular reflection from roughened surfaces," Journal of Optical Society of America, 57(9):1105-1114, 1967.

[9] M. Ashikhmhin and P. Shirley. "An anisotropic phong BRDF model," Journal of Graphics Tools, 5(2):25-32, 2000.

[10] Y. Dong, J. Wang, X. Tong, J. Snyder, Y Lan, M. BenEzra, B. Guo, "Manifold Bootstrapping for SVBRDF Capture,"ACM Transactions on Graphics Volume 29, Number 4, July 2010.
[11] T. Morimoto, R. T. Tan, R. Kawakami, and K. Ikeuchi, "Estimating optical properties of layered surfaces using the spider model," Proc. CVPR2010.

[12] K. Iwasaki, K. Matsuzawa, and T. Nishita, "Real-time rendering of soap bubbles taking into account light interference," Computer Graphics International, pp. 344-348, 2004.

[13] H. Hirayama, K. Haneda, H. Yamashita, and Y. Monden, "An accurate illumination model for objects coated with multilayer films," Eurographics, pp. 145$150,2000$.

[14] H. Hirayama, Y. Yamaji, K. Kaneda, H. Yamashita, and Y. Monden, "Rendering iridescent colors appearing on natural objects," Pacific Graphics, p. 15, 2000.

[15] Y. Sun, F. D. Fracchia, M. S. Drew, and T. W. Calvert, "Rendering iridescent colors of optical disks," Eurographics Workshop on Rendering Techniques, pp. 341$352,2000$.

[16] Y. Sun, F. D. Fracchia, T. W. Calvert, and M. S. Drew, "Deriving spectra from colors and rendering light interference," Computer Graphics and Applications, Vol. 19, pp. 61-66, 1999.

[17] I. Sadeghi, A. Munoz, P. Laven, W. Jarosz, F. Seron, D. Gutierrez, H. W. Jensen, "Physically-based Simulation of Rainbows," ACM Trans.Graph., Vol 31(1), pp.3:1?3:12, 2012.

[18] T. Cuypers, S.B. Oh, T. Haber, P. Bekaert, R. Raskar, "Reflectance Model for Diffraction," ACM Trans. Graph., Vol 31 (5), pp. 1-11, 2012.

[19] K. W. Meissner, "Interference Spectroscopy. Part I," Journal of Optical Society of America, Vo; 31 (6), pp. 405-427, 1941.

[20] R. M. A. Azzam, N. M. Bashara, "Ellipsometry and Polarized Light," Elsevier Science Pub Co, 1987.

[21] S. Kinoshita, "Structural Colors in the Realm of Nature," World Scientific Pub Co Inc , 2009.

[22] Matt Pharr and Greg Humphreys, "Physically Based Rendering from Theory to Implementation," Morgan Kaufmann, 2004. 